\title{
LA AGENDA TEMÁTICA EN LA PRENSA DE LOS PAÍSES MEDITERRÁNEOS, LA APORTACIÓN DE DERECHOS Y LA CREACIÓN DE ESTADOS DE OPINIÓN ${ }^{1}$
}

\author{
THE AGENDA SETTING IN THE PRESS OF THE \\ MEDITERRANEAN COUNTRIES, THE CONTRIBUTION OF \\ RIGHTS AND THE CREATION OF OPINION STATES
}

\author{
Ricardo Carniel Bugs y Teresa Velázquez García-Talavera*
}

Recibido: 18/01/2017

Aceptado: 14/03/2017

SUMARIO: I. INTRODUCCIÓN. II. LOS DIARIOS OBJETO DE ESTUDIO Y LA MUESTRA DE ANÁLISIS. III. LA TOMA DE DECISIÓN EN LOS DIARIOS ANALIZADOS. IV. ¿CUÁLES SON LOS TEMAS PRESENTES EN LOS DIARIOS ANALIZADOS Y CUÁL SU ENFOQUE? LA AGENDA TEMÁTICA. V. LOS DERECHOS HUMANOS COMO ELEMENTOS DE AGENDA VI. PAÍSES REFERENCIADOS EN LAS UNIDADES DE ANÁLISIS Y SU VINCULACIÓN CON LAS RELACIONES MULTILATERALES Y BILATERALES. VII. LOS ACTORES PRESENTES EN LAS UNIDADES DE ANÁLISIS. VIII. CONCLUSIONES. IX. BIBLIOGRAFÍA.

SUMMARY: I. INTRODUCTION. II. THE MEDIA OBJECT OF STUDY AND THE SAMPLE OF ANALYSIS. III. THE DECISION MAKING IN THE ANALYZED NEWSPAPERS. IV. WHAT ARE

\footnotetext{
1 Parte del contenido de esta aportación fue presentado en el Área Temática 4. Opinión Pública, Comunicación y Medios, del XII Congreso Nacional y V Congreso Internacional sobre Democracia, organizado por la Facultad de Ciencia Política y Relaciones Internacionales de la Universidad Nacional de Rosario. y celebrado en Rosario (Argentina), durante los días del 12 al 15 de septiembre de 2016.

\begin{abstract}
* Ricardo Carniel es Doctor en Ciencias de la Comunicación (Periodismo) por la UAB y profesor del Departamento de Periodismo y Ciencias de la Comunicación de la UAB. Coordinador académico adjunto en la UAB del Máster Erasmus Mundus "Crossing Mediterranean: towards investment and Integration/Mediación Intermediterránea: hacia la invesrisón y la integración (MIM)” impartido por la UAB, y las Universidades Ca'Foscari de Venecia y Montpellier III. Investigador del LAPREC, Grupo de investigación consolidado (2014 SGR 1388) de la Generalitat de Catalunya en la UAB. (ricardo.carniel@uab.cat)

Teresa Velázquez es catedrática de Periodismo en la UAB, ámbito de teoría, semiótica y metodología de investigación en comunicación. Investigadora Principal del proyecto "La construcción social del espacio intermediterráneo y sus correlaciones en la agenda temática de los medios de comunicación, La información en prensa y televisión” (CSO2012-35955), Ministerio español de Economía y Competitividad (2013-2016), desarrollado por el Laboratori de Prospectiva i Recerca en Comunicació, Cultura i Comunicació (LAPREC), Grupo de investigación consolidado (2014 SGR 1388) de la Generalitat de Catalunya en la UAB, del que es directora. Es presidenta del Observatorio Mediterráneo de la Comunicación (OMEC). Coordinadora académica del Módulo de Barcelona del Máster Erasmus Mundus "Crossing Mediterranean: towards investment and Integration/Mediación Intermediterránea: haciala inversión y la integración (MIM)" impartido por la UAB, y las Universidades Ca’Foscari de Venecia y Montpellier III. (teresa.velazquez@uab.cat)
\end{abstract}


THE TOPICS PRESENT IN THE ANALYZED NEWSPAPERS AND THEIR APPROACH? THE THEMATIC AGENDA. V. THE HUMAN RIGHTS AS ELEMENTS OF AGENDA VI. COUNTRIES REFERENCED IN THE UNITS OF ANALYSIS AND THEIR LINKAGE TO MULTILATERAL AND BILATERAL RELATIONS. VII. THE ACTORS PRESENT IN THE UNITS OF ANALYSIS. VIII. CONCLUSIONS IX. BIBLIOGRAPHY.

Resumen: La información presentada y difundida por los medios de comunicación establece una correlación entre la forma de expresión-noticia y la creación de estados de opinión. Esta contribución presentará resultados parciales de la prensa de países mediterráneos

durante la primera semana de mayo de 2013, de la investigación "La construcción social del espacio intermediterráneo y sus correlaciones en la agenda temática de los medios de comunicación, La información en prensa y televisión” (CSO2012-35955), Ministerio español de Economía y Competitividad (2013-2016). El tratamiento sobre relaciones multi y bilaterales, política y legislación, paz y seguridad, economía y finanzas, derechos humanos se aborda mediante el análisis de contenido aplicado a diarios de Argelia, Egipto, Marruecos, Túnez, España, Francia, Italia y Reino Unido.

Palabras clave: Agenda temática; prensa países mediterráneos; derechos humanos; opinión pública

Abstract: The information presented and disseminated by the media establishes a correlation between the form of expression-news and the creation of states of opinion. This contribution will present partial results from the press of Mediterranean countries during the first week of May 2013, from the research "The social construction of the intermediterranean space and its correlations in the thematic agenda of the media, Information in press and television" (CSO2012-35955), Spanish Ministry of Economy and Competitiveness (2013-2016). The treatment of multi and bilateral relations, politics and legislation, peace and security, economy and finance, human rights is addressed through content analysis applied to newspapers in Algeria, Egypt, Morocco, Tunisia, Spain, France, Italy and the United Kingdom.

Keywords: Thematic agenda; Press Mediterranean countries; human rights; public opinion

\section{I.INTRODUCCIÓN}

La información presentada y difundida por los medios de comunicación guarda una estrecha relación con las propuestas de lectura contenidas en la construcción de dicha información. La correlación entre la forma de expresión-noticia y la creación de estados de opinión, como primer estadio de Opinión Pública, son aspectos que deben ser considerados en correlación. 
Esta exposición presentará resultados parciales de análisis de la prensa de países mediterráneos correspondiente a la primera semana de mayo de 2013, extraídos de la investigación titulada "La construcción social del espacio intermediterráneo y sus correlaciones en la agenda temática de los medios de comunicación. La información en prensa y televisión” (CSO2012-35955) ${ }^{2}$, financiada por el Ministerio de Economía y Competitividad (MINECO) español (2013-2016).

prensa y televisión” (CSO2012-35955) ${ }^{3}$, financiada por el Ministerio de Economía y Competitividad (MINECO) español (2013-2016).

Entre los diarios observados se encuentran Assabah (Marruecos), Al-Dustour (Egipto), Attounissia (Túnez), El Watan (Argelia), ABC (España), Le Monde (Francia), La Repubblica (Italia), The Times (Reino Unido) y Attounissia (Túnez)

Las relaciones multi y bilaterales, política y legislación, paz y seguridad, economía y finanzas, derechos humanos, entre otros temas, se abordan mediante el análisis de contenido. Determinar quiénes son los actores institucionales, sociales o los individuos destacados en estas informaciones es otro de los aspectos a presentar.

\section{II.LOS DIARIOS OBJETO DE ESTUDIO Y LA MUESTRA DE ANÁLISIS}

Los diarios estudiados por país y las unidades de análisis en cada uno de ellos, son: Argelia (El Watan, 56); Egipto (Al Dustour, 76); España (ABC, 74); Francia (Le Monde, 97); Italia (La Repubblica, 54); Marruecos (As Sabah, 110); Túnez (Attounissia, 153), Reino Unido (The Times, 40).

La muestra quedó constituida en 660 casos para estos diarios y durante esa primera semana.

\footnotetext{
2 Investigadora Principal, Teresa Velázquez García-Talavera. Investigadores por orden alfabético: Hesham Abu-Sharar, Maria Luz Barbeito Veloso, Zhour Bouzidi, Ricardo Carniel Bugs, Carolina CerdaGuzman, Lluís Costa Fernández, Carmina Crusafon Baques, Olga Del Río Sánchez, Lucrecia Escudero Chauvel, Natalia Fernández Díaz, Elisabet Garcia Altadill, Juan Antonio García Galindo, Víctor Gutiérrez Castillo, Nouredinne Harrami, Gabriel Lemkow Tovias (desde enero 2015), Carmen Martínez Romero, Patria Roman Velázquez, Fernando Sabés Turmo (hasta el 17/06/2015), Susana Tovias Wertheimer (hasta el 03/11/2014), José Juan Verón Lassa. Profesor invitado adscrito al proyecto: Amilton Gláucio de Oliveira (desde 30/10/2014-3010/2015). Doctorandos adscritos al proyecto: Estibaliz Ortega Miranda (FPI), José Miguel Gámez Pérez, Hicham El Bakouti, Hanane Ashina, Valentina Saini.

3 Investigadora Principal, Teresa Velázquez García-Talavera. Investigadores por orden alfabético: Hesham Abu-Sharar, Maria Luz Barbeito Veloso, Zhour Bouzidi, Ricardo Carniel Bugs, Carolina CerdaGuzman, Lluís Costa Fernández, Carmina Crusafon Baques, Olga Del Río Sánchez, Lucrecia Escudero Chauvel, Natalia Fernández Díaz, Elisabet Garcia Altadill, Juan Antonio García Galindo, Víctor Gutiérrez Castillo, Nouredinne Harrami, Gabriel Lemkow Tovias (desde enero 2015), Carmen Martínez Romero, Patria Roman Velázquez, Fernando Sabés Turmo (hasta el 17/06/2015), Susana Tovias Wertheimer (hasta el 03/11/2014), José Juan Verón Lassa. Profesor invitado adscrito al proyecto: Amilton Gláucio de Oliveira (desde 30/10/2014-3010/2015). Doctorandos adscritos al proyecto: Estibaliz Ortega Miranda (FPI), José Miguel Gámez Pérez, Hicham El Bakouti, Hanane Ashina, Valentina Saini.
} 
Estos diarios cuentan con una amplia circulación en los países en los que se publican por lo que hemos considerado de interés seleccionarlos.

Acotaremos en primer lugar, los aspectos estructurales y de jerarquización de las informaciones y las categorías en las que nos fijamos, como son la selección de la

información, en otras palabras, qué es lo que lleva a los medios de comunicación a priorizar unos acontecimientos sobre otros. Una vez hecha la selección, qué es lo que se jerarquiza y cuál es el lugar que ocupa el tema objeto de observación en el espacio del diario. En esta selección y jerarquización de temas se construye la agenda temática, a partir de la cual se generará el proceso de tematización que da lugar al debate público de los temas de interés público.

La selección de los temas a observar responde a aquellos objetivos y recomendaciones de instancias supranacionales como son Unión por el Mediterráneo (UpM), surgida del Proceso de Barcelona, la política Europea de Vecindad (PEV) y la Alianza de Civilizaciones (AC)

Señalamos a continuación aquellos aspectos que hemos encontrado más referenciados en la muestra objeto de estudio y que se encuentran extraídos de los objetivos y recomendaciones de estas instancias supranacionales, las cuales destacan dentro de Política y legislación aspectos vinculados a Derecho internacional y derechos humanos, Democracia y buena gobernanza, Partidos políticos, Integridad regional y/o autodeterminación pueblos, Políticas multilaterales /bilaterales, Liderazgo político responsable. Respecto a Paz y seguridad, por ejemplo, algunos valores se centran en Conflicto armado, Conflicto diplomático, Terrorismo nacionalista/independentista, Terrorismo internacional, Delincuencia organizada, Migración irregular, Cooperación militar y/o policial, Promoción de la paz y la convivencia o Ayuda humanitaria o, en el caso de Economía y finanzas, Zona euromediterránea de libre comercio, Recursos energéticos, Recursos naturales y medio ambiente, Desarrollo sostenible, Infraestructuras y transportes, Integración económica Sur - Sur, Reformas financieras, Inversiones extranjeras/ Fomento de inversiones, Fomento de la inversión nacional, Pobreza y/o desigualdades económicas, Crisis económica (mundial o global), Cooperación al desarrollo, Cooperación empresarial/industrial. Los resultados obtenidos en esta investigación referidos a la presencia de estos temas en la muestra analizada se pueden ver en el Gráfico 10, apartado 3 de esta presentación.

La parte del trabajo referida al tratamiento de los contenidos consiste en un análisis cuantitativo de contenido sobre las noticias aparecidas en el periodo seleccionado referidas a los temas que hemos indicado en la introducción de esta presentación. El procedimiento seguido ha sido el habitual en la aplicación de esta técnica. Lo que sí nos parece oportuno hacer constar está en relación con los ámbitos en los que quedó dividido el instrumento de análisis y que nos han servido para la extracción de datos. Así, encontramos categorías de carácter descriptivo y de toma de decisión (extensión, 
orden, proporción, sección, fuentes, etc.) y otras relacionadas con los temas y su ponderación.

Pasemos a presentar la presencia de informaciones en torno al tema que nos ocupa por diarios:

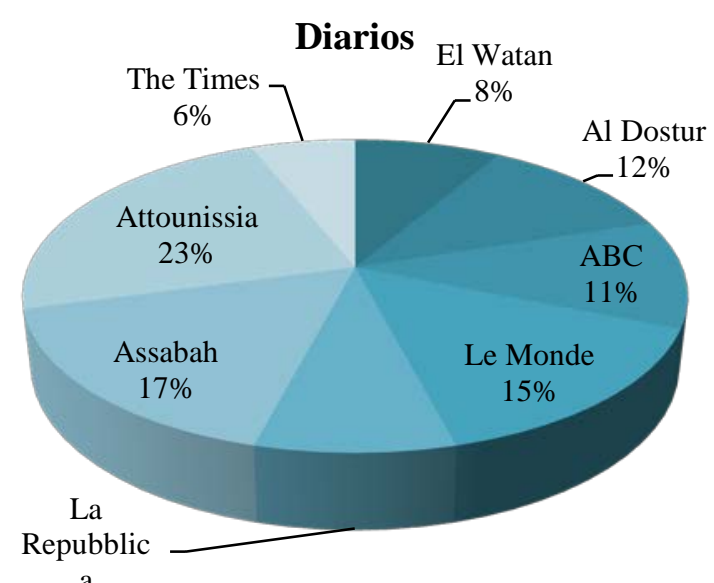

Gráfico 1. Fuente: elaboración propia a partir de los datos extraídos y analizados en la investigación

\section{III.LA TOMA DE DECISIÓN EN LOS DIARIOS ANALIZADOS.}

Pasemos ahora a exponer aquellos aspectos vinculados a la toma de decisión de manera general. Los elementos que consideramos para ello se relacionan con los aspectos estructurales. Así, si las unidades de análisis han sido destacadas y en dónde, qué extensión los diarios dan a estas informaciones, dónde se ubican, a qué género informativo responden, en qué sección del medio aparecen y si van acompañadas de imagen o no.

Las figuras profesionales para llevar a cabo estas tareas las constituyen el Gatekeeper o seleccionador y el Newsmaking o la verificación y ampliación de la información. El primero de ellos, y dentro de las rutinas profesionales, se refiere a la función del periodista como seleccionador de los hechos que acontecen y los criterios que aplica a su selección. Por lo tanto, relacionado con la "Agenda setting” resultante (que veremos en el apartado siguiente), con la toma de decisiones y, en consecuencia, esta rutina profesional favorecerá la circulación de la información, entre otros, dentro de los grupos sociales primarios, de este modo se relaciona con lo influencia de los medios.

La segunda figura señalada, el Newsmaking, tiene por función la verificación y ampliación de la información. Éste es otro aspecto que se encuentra dentro de las rutinas profesionales, los medios como organizaciones institucionales y la cultura profesional de los periodistas, y responde a un proceso jerárquicamente ordenado, vinculado a conceptos tales cómo, noticiabilitad, relevancia, eficiencia, impacto, proximidad, 
rapidez. El formato, como manera de presentar la información, también es determinante. En esta etapa de las rutinas profesionales se verifica, amplía e interpreta la información.

En la serie de gráficos que aparecen a continuación se apreciarán estos aspectos vinculados a la toma de decisión y a las rutinas profesionales:

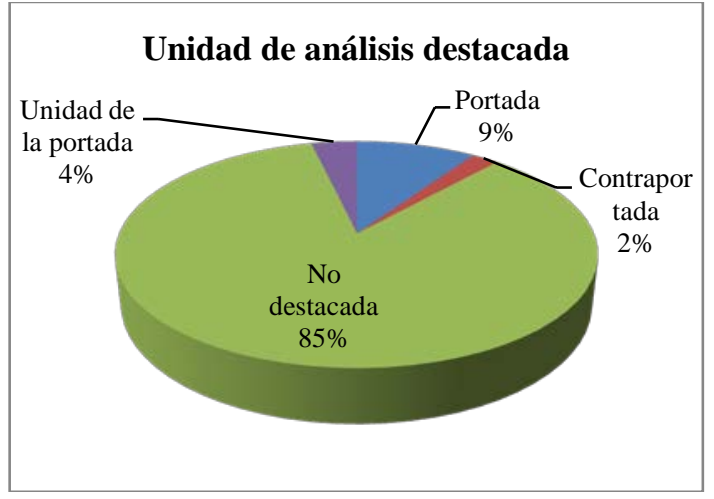

Gráfico 2

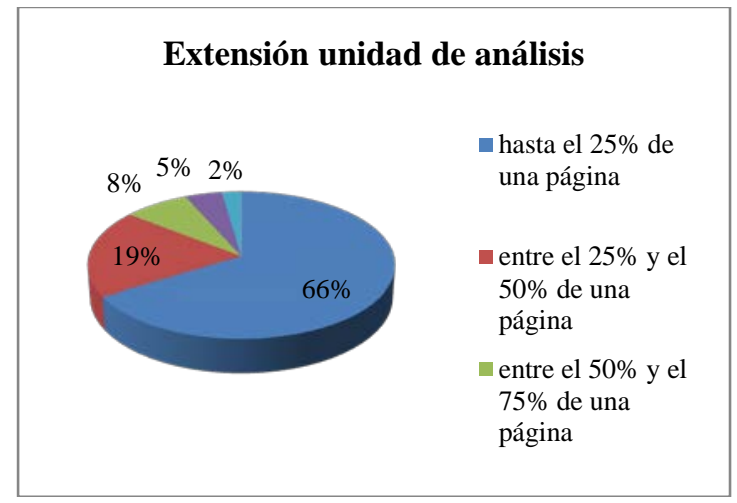

Gráfico 3

Fuente gráficos 2 y 3: Fuente: elaboración propia a partir de los datos extraídos y analizados en la investigación

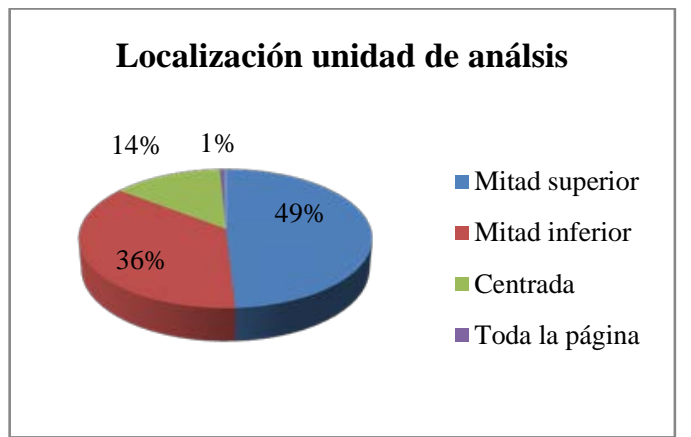

Gráfico 4

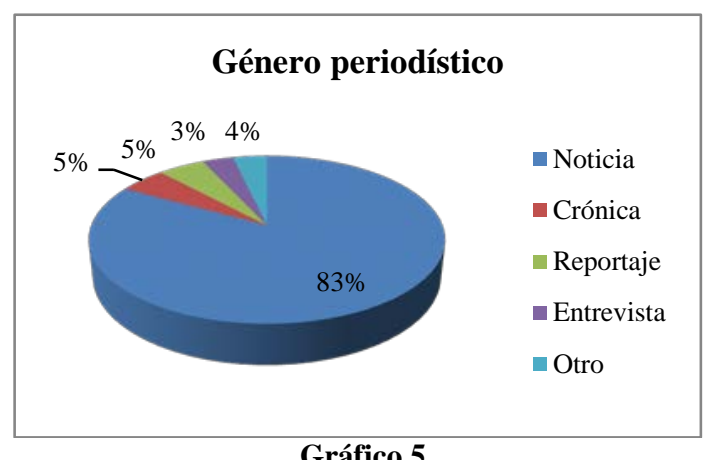

Gráfico 5

Fuente gráficos 4 y 5: elaboración propia a partir de los datos extraídos y analizados en la investigación 
La agenda temática en la prensa de los países mediterráneos, la aportación de derechos y la creación de estados de opinión

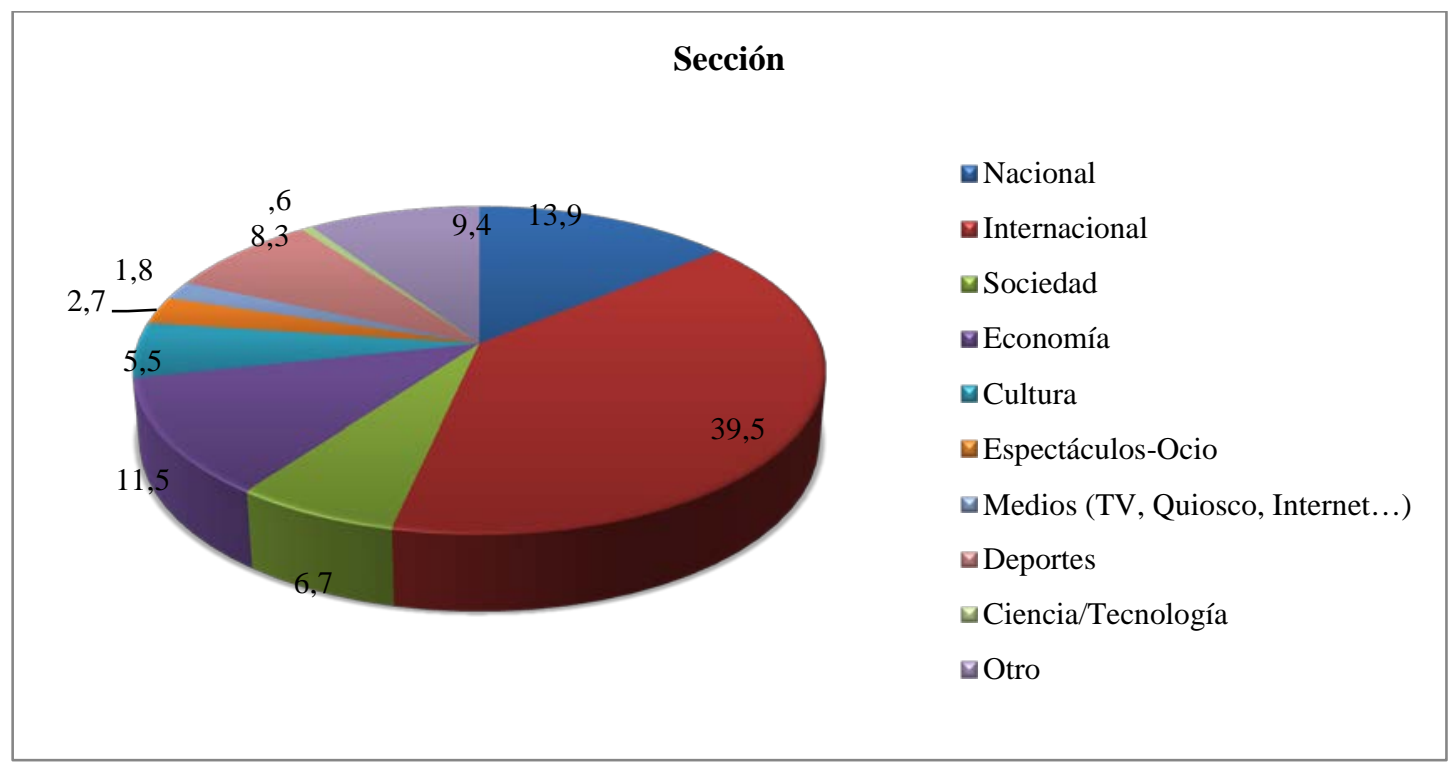

Gráfico 6. Fuente: elaboración propia a partir de los datos extraídos y analizados en la investigación La agenda temática en la prensa de los países mediterráneos, la aportación de derechos y la creación de estados de opinión

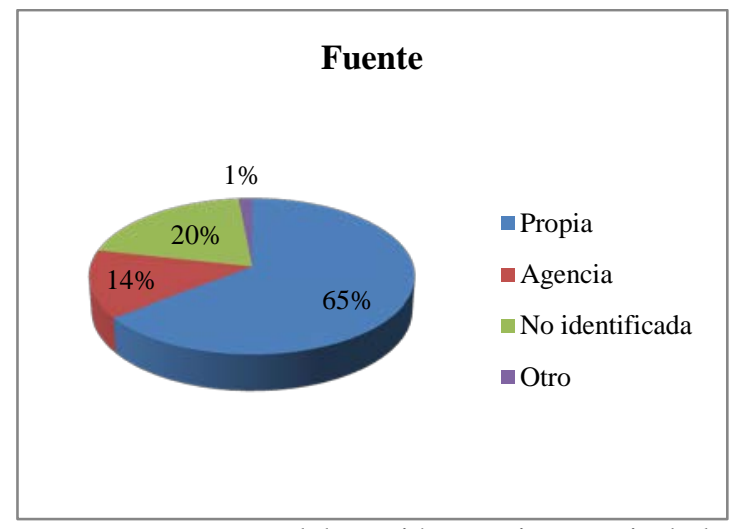

Gráfico 7. Fuente: elaboración propia a partir de los datos extraídos y analizados en la investigación

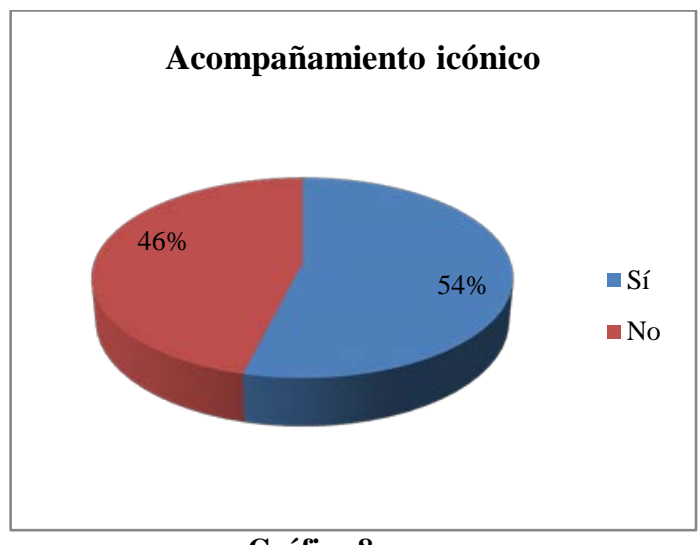

Gráfico 8

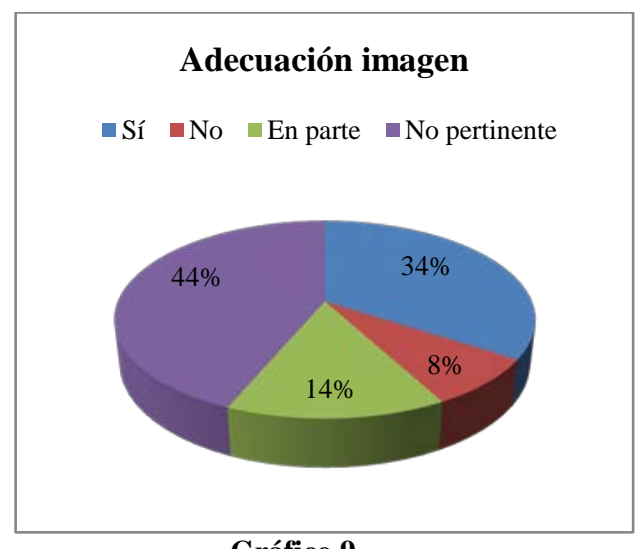

Gráfico 9

Fuente de los gráfico 8 y 9: elaboración propia a partir de los datos extraídos y analizados en la investigación 
Estos resultados nos indican que los medios de comunicación analizados no destacan las informaciones sobre los temas propuestos. Hecho manifiesto en el 85\% de las informaciones analizadas. Este hecho queda corroborado con la extensión que le adjudican, dado que el $66 \%$ se corresponden a aquellas informaciones que ocupan hasta un $25 \%$ de una página. Sin embargo este aspecto se corrige al ubicar un $49 \%$ en la mitad superior, espacio en el que los medios sitúan aquellas informaciones que consideran de interés.

En cuanto al género periodístico más utilizado éste se corresponde a la noticia y respecto a la sección, el mayor porcentaje recae en internacional. Ante esta evidencia, es interesante conocer las fuentes de información de las que se nutre el diario y vemos que, mayoritariamente son fuentes propias. Este hecho indicaría una presencia mediante corresponsales de los medios en los lugares de los acontecimientos. Realmente, esta evidencia nos sorprende.

Otro elemento que nos parece importante destacar es si estas informaciones van acompañadas de imágenes y si éstas muestran coherencia con el total de la información. Los resultados indican que en un 54\% de los caso, las informaciones cuentan con acompañamiento icónico de los cuales, un 34\% se ajusta, un 8\% no, y un 14\% en parte. Quiere decir que este aspecto es cuidado por los diarios.

En líneas generales podemos deducir que los diarios analizados durante el periodo de la muestra manifiestan una toma de decisión bastante ponderada.

\section{IV. ¿CUÁLES SON LOS TEMAS PRESENTES EN LOS DIARIOS ANALIZADOS Y CUÁL SU ENFOQUE? LA AGENDA TEMÁTICA}

Sabemos que la actividad profesional periodística da como resultado la contribución de los medios de comunicación a la construcción social de la realidad. En este sentido, y desde nuestra opinión, estamos convencidos que la prensa, sea en formato off line, como on line, mantiene un papel determinante en este sentido.

También, hay que hacer constar la responsabilidad social que los medios de comunicación en general, y la prensa en particular, tienen respecto a su influencia en la ciudadanía, puesto que son actores institucionalizados y legitimados. Ahí radica su responsabilidad social.

En este sentido, la información, en concreto la noticia, se ha institucionalizado (Tuchman, 1983) en la sociedad como el relato "objetivo" de los acontecimientos. La institución da carácter público a los hechos y éstos son importantes para la vida pública social. 
La noticia propone y construye una realidad a partir de las rutinas profesionales, tal y como hemos indicado en el apartado anterior, pero no es un reflejo de la realidad, sino una realidad construida, pública y desde un punto de vista. Por lo tanto, responde a una forma de conocer la realidad según el periodista. Este aspecto estará vinculado a la información como contribución a la construcción social de la realidad y, por lo tanto, como influencia a largo plazo y sobre el conocimiento.

Veremos como estos aspectos se encuentran íntimamente vinculados a la creación de opinión pública sobre la que volveremos más adelante.

Los medios influyen en el conocimiento de la realidad y son instituciones con poder en la sociedad. La información es, como acabamos de ver, un elemento clave dentro de los efectos sobre el conocimiento.

Además, los medios confieren status a aquellos que aparecen en los mismos y mantienen el status quo, aspecto que desarrollaremos más adelante

Hechas estas precisiones concretemos que entendemos por Agenda setting ${ }^{4}$ y cómo ésta se encuentra presente en nuestro trabajo.

El proceso del establecimiento de la Agenda temática sigue los siguientes pasos: a) selección por parte del medio de los hechos que considera más relevante (teorías del Gatekeeper y Newsmaking ya indicadas en el apartado anterior de este texto). Estos hechos constituyen los temas a tratar y, por lo tanto, aquellos que se transformaran en acontecimientos. Este paso del proceso será un elemento importante para el conocimiento que la gente tendrá sobre la realidad social, también, está relacionado con la toma de decisión; b) jerarquización ${ }^{5}$, a partir de la selección, el medio establecerá un orden de relevancia sobre los temas, es decir, de qué manera aparecerán, serán tratados, bajo qué forma de expresión o género, en qué lugar del medio. Todo ello relacionado, también, con la toma de decisión (teorías de los Gatekeeper y del Newsmaking), vinculada, tal y como hemos expuesto, a la teoría de los efectos a largo plazo o sobre el conocimiento. (Franco Rositi, 1982; Mauro Wolf, 1994 )

A partir del proceso anterior, los medios contribuyen al debate público de los temas de interés público. Esto es lo que se denomina "tematización” y constituye uno de los aspectos de los efectos a largo plazo o sobre el conocimiento. (Carlo Marletti, 1985; Giorgio Grossi, 1984, 2007). Este aspecto tiene vital importancia para la creación de Opinión pública, la cual, y a partir de los géneros informativos pone en marcha el primer estadio de opinión pública, denominado los estados de opinión.

\footnotetext{
${ }^{4}$ Este concepto fue establecido por Maxwell McCombs y Donald Shaw en su artículo titulado "The agenda-setting function of mass media", publicado en Public Opinion Quarterly, 36 (2): 176, en 1972. Una actualización de esta teoría se encuentra en McCombs (2014).

${ }^{5}$ Véase Gráficos del 2 al 6 en el apartado anterior
} 


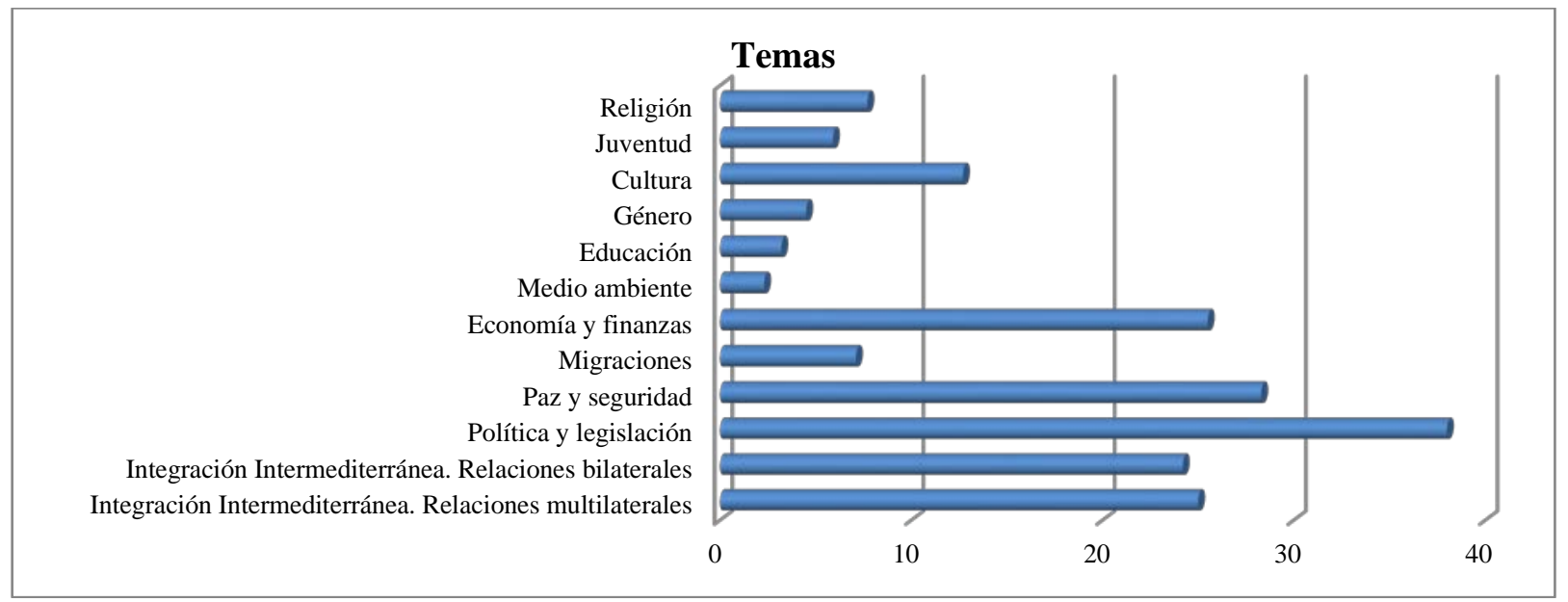

Gráfico 10

Fuente: elaboración propia a partir de los datos extraídos y analizados en la investigación

\section{Presencia/ausencia de temas}

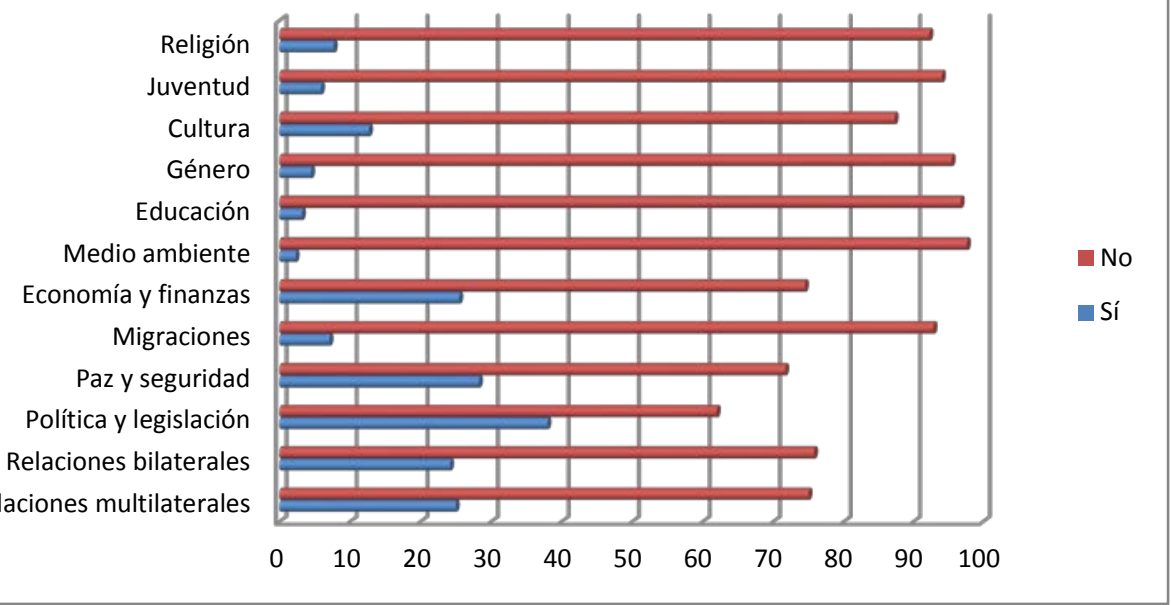

Gráfico 11

Fuente: elaboración propia a partir de los datos extraídos y analizados en la investigación

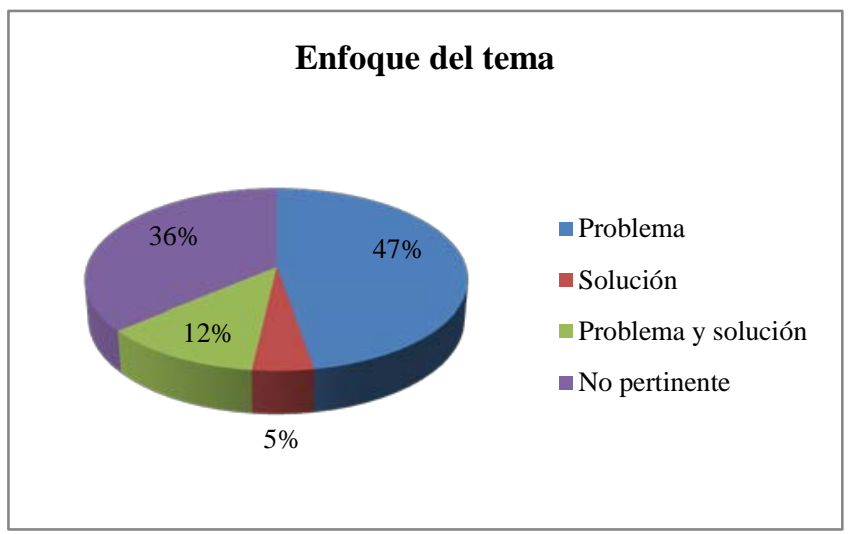

Revista de Estudios Jurídicos n 17/2017 (Segunda Época)

ISSN-e 2340-5066. Universidad de Jaén (España)

Versión electrónica: rej.ujaen.es 
Gráfico 12
Fuente: elaboración propia a partir de los datos extraídos y analizados en la investigación

Estos gráficos indican que los temas más tratados se relacionan con Política y Legislación (38\%), Paz y seguridad (28,3) y Economía y finanzas (25,5\%), al margen de las Relaciones multilaterales (25\%) y bilaterales $(24,2 \%)$ que desarrollaremos en el apartado siguiente.

Estos temas forman parte de la contribución a la construcción social de la realidad que el medio prensa analizado efectúa sobre los aspectos de interés vinculados a los países mediterráneos. Destaca, también, que el enfoque de los temas en general responde a problema (47\%), frente a un mínimo 5\% como solución y un 12\% de problema y solución.

Creemos que estos resultados responden a la situación geopolítica, y también a las transformaciones que se producen en el espacio mediterráneo.

\section{LOS DERECHOS HUMANOS COMO ELEMENTOS DE AGENDA}

Interesa destacar la presencia de los Derechos Humanos en la agenda de los medios objeto de análisis.

Tomamos en consideración cuando en la muestra analizada hay mención o referencias a Derechos civiles y políticos, Derechos económicos, sociales y culturales, Derechos de los pueblos y Derechos a la información, comunicación y ciberespacio y dentro de ello, los valores que se asocian respecto a su vulneración o violación o a su promoción o protección.

Respecto a estas caracterizaciones, la presencia de los DD.HH. responde a un total de 221 unidades de análisis, lo que supone un 35,5\%

En cuanto a si la vulneración o violación aparece explícitamente recogida, su presencia es de 50 unidades de análisis, lo que supone un 7,6\%, mientras que en el caso de si ésta es implícita, encontramos 93 unidades, lo que supone un 14,1\%

En lo referente a la promoción o protección de los derechos explícitamente referida, encontramos 19 casos, un 2,9\%, mientras que implícitamente aparece en 59 unidades, lo que supone un $8,9 \%$

Agrupados podemos observar que la vulneración o violación, sea ésta implícita o explícita, supone un $21,7 \%$, frente a la promoción y protección que supone un $11,8 \%$ de total. 


\section{Referencia a Derechos Humanos}

No hay referencia explícita o implícita

Implícitamente Promoción / protección

Explícitamente Promoción / protección

Implícitamente Vulneración / violación

Explícitamente Vulneración / violación

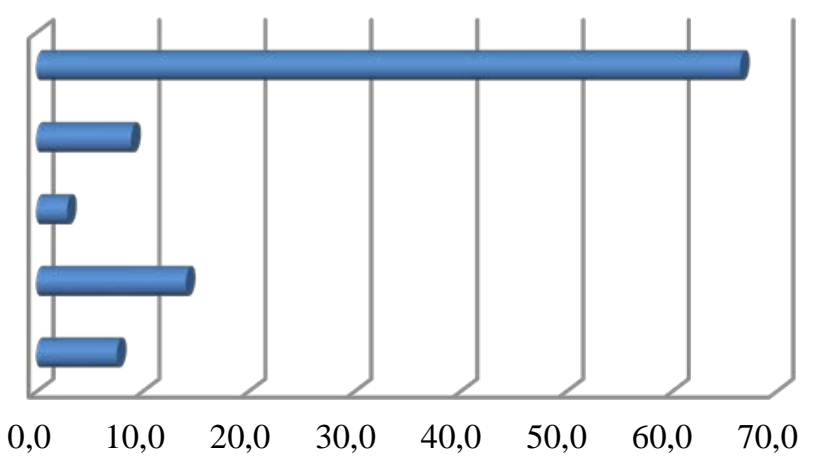

Gráfico 13

Fuente: elaboración propia a partir de los datos extraídos y analizados en la investigación

Estos resultados nos indican que el tema de los DD.HH. es información cuando ésta se relaciona con la vulneración de los mismos.

VI. PAÍSES REFERENCIADOS EN LAS UNIDADES DE ANÁLISIS Y SU VINCULACIÓN CON LAS RELACIONES MULTILATERALES Y BILATERALES.

Si el espacio mediterráneo es considerado un espacio estratégico y de interés para todos los países que lo circundan, creemos que la presencia de diferentes países en las informaciones analizadas, así como, el tipo de relaciones que se establecen entre ellos son de interés para responder y evidenciar la denominada Integración Intermediterránea.

Para referirnos a este tema, partimos de la noción geográfica en la distinción Norte/Sur, por lo tanto, no se toma en cuenta el grado de desarrollo de los países.

En lo que respecta a nuestra investigación los resultados quedan reflejados en el gráfico que presentamos a continuación 


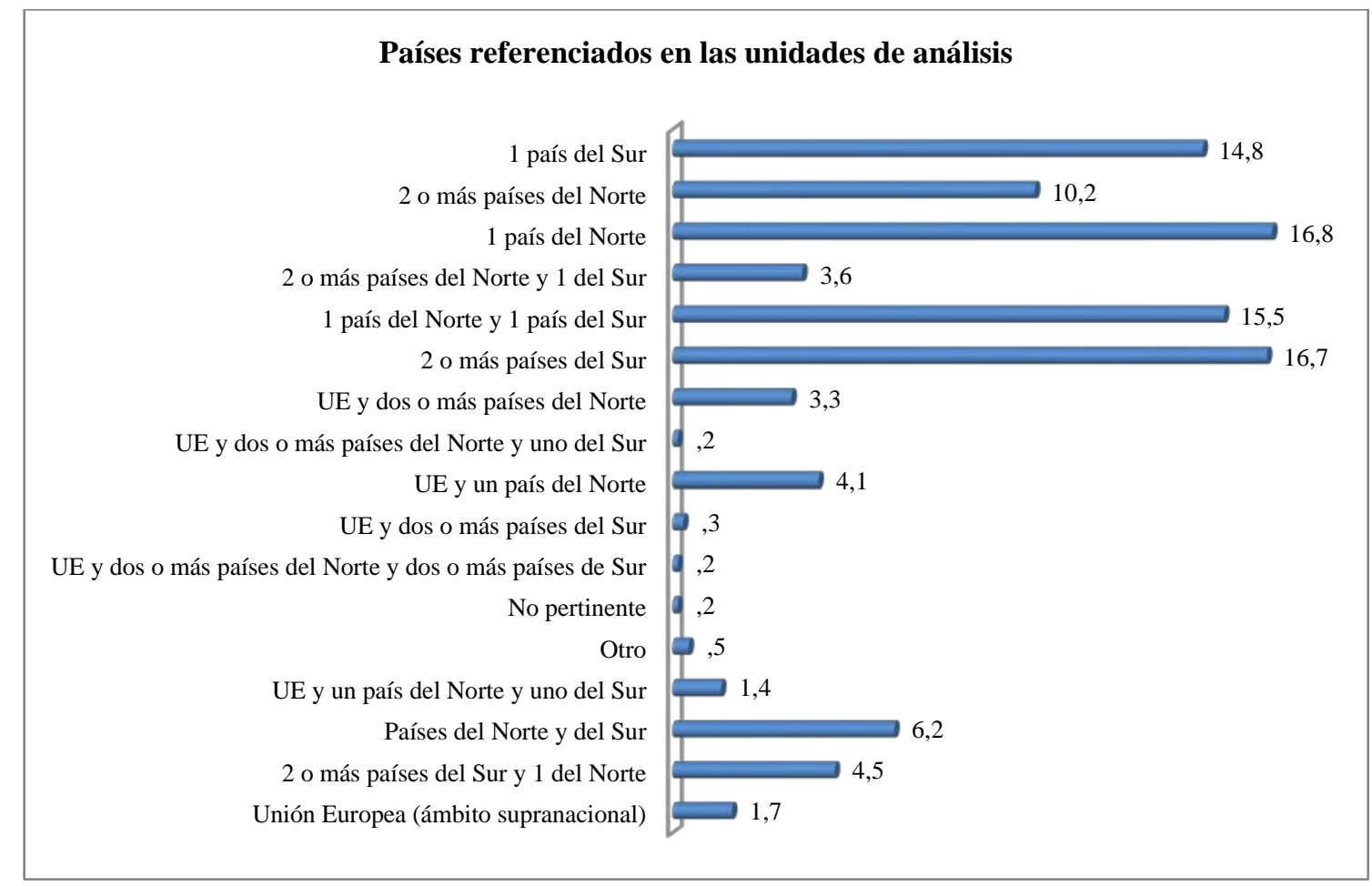

\section{Gráfico 14}

Fuente: elaboración propia a partir de los datos extraídos y analizados en la investigación

Vemos que cuando en las informaciones se referencian varios países del Sur, su presencia es de un 16,7\% lo que unido al resultado de un 14,8\% de aparición de un país del Sur, ello supone un 31,5\%.

En cuanto a países del Norte, se constata un 16,8\% para un país del Norte, unido a un $10,2 \%$ de dos o más países del Norte, el resultado es de un $27 \%$

En cuanto a la presencia de países del Norte y del Sur, encontramos los siguientes resultados: para un país del Norte y uno del Sur (15,5\%); para dos o más países del Norte y uno del Sur (3,6\%); Países del Norte y del Sur (6,2\%); dos o más países del Sur y uno del Norte $(4,5)$. El total de estas relaciones es de un $29,8 \%$

Expuestos estos resultados, veamos ahora como se concretan los mismos en la Integración intermediterránea y sus políticas multilaterales y bilaterales. 


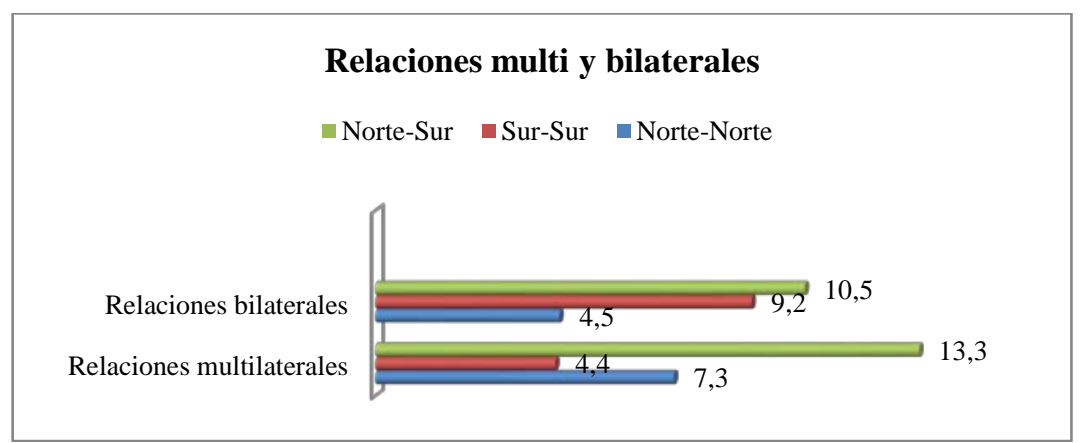

\section{Gráfico 15}

Fuente: elaboración propia a partir de los datos extraídos y analizados en la investigación

Lo que nos indican estos resultados es que tanto en las relaciones multilaterales, como bilaterales, las que más predominan son las Norte-Sur.

Dentro de estas relaciones Norte-Sur, nos fijaremos en cómo se concretan cuando las cruzamos con los temas con mayor peso porcentual y que se corresponden con Política y legislación, Paz y seguridad y Economía y finanzas.

En primer lugar expondremos resultados sobre relaciones multilaterales vinculadas a los temas mencionados. Así, en Política y legislación destaca Derecho internacional y derechos humanos, con un $8 \%$ en las relaciones Norte-Sur.

En cuanto a Paz y seguridad, destaca Conflicto armado, con un 8\%; terrorismo internacional, con un 6,8\%; Cooperación militar y/o policial, con un 4,5\%; finalmente para Promoción de la paz y la convivencia nos encontramos con un 11,4\%.

Para Economía y finanzas, lo más destacado es Inversiones extranjeras/ Fomento de inversiones, con un $6,8 \%$

Los resultados correspondientes a las relaciones bilaterales entre el Norte y el Sur y en relación a los temas destacados, encontramos que, para Política y legislación, el valor más destacado es, también, Derecho internacional y derechos humanos, con un 10,3\%.

\section{Ricardo Carniel y Teresa Velázquez}

En relación a Paz y seguridad, los valores más destacados son: Delincuencia organizada, con un 29,4\%; Migración irregular, con un 28,6\%; Conflicto diplomático, con un 27,3\%; Terrorismo nacionalista/independentista, con un 20\%; Promoción de la paz y la convivencia, con un 16\%; Cooperación militar y/o policial, con un10\%.

En cuanto a Economía y finanzas, para las relaciones bilaterales Norte-Sur, destaca para Zona Euromediterránea de libre comercio (1,4\%), Recursos energéticos (1,4\%), Infraestructuras y transporte (1,4\%), Cooperación al desarrollo, $(2,4 \%)$ si bien estos resultados son pequeños, indican que estos son los intereses de los países destacados por los medios estudiados respecto a las relaciones bilaterales entre el Norte y el Sur. 


\section{LOS ACTORES PRESENTES EN LAS UNIDADES DE ANÁLISIS}

Como indicábamos en el apartado IV los medios de comunicación confieren status a aquellos que aparecen en los mismos.

Creemos importante señalar qué actores son los que aparecen destacados en el titular y el lid de las unidades analizadas y cuál es su status y rol.

El gráfico siguiente ilustra la ponderación de los diferentes tipos de actores que aparecen destacados en las informaciones.

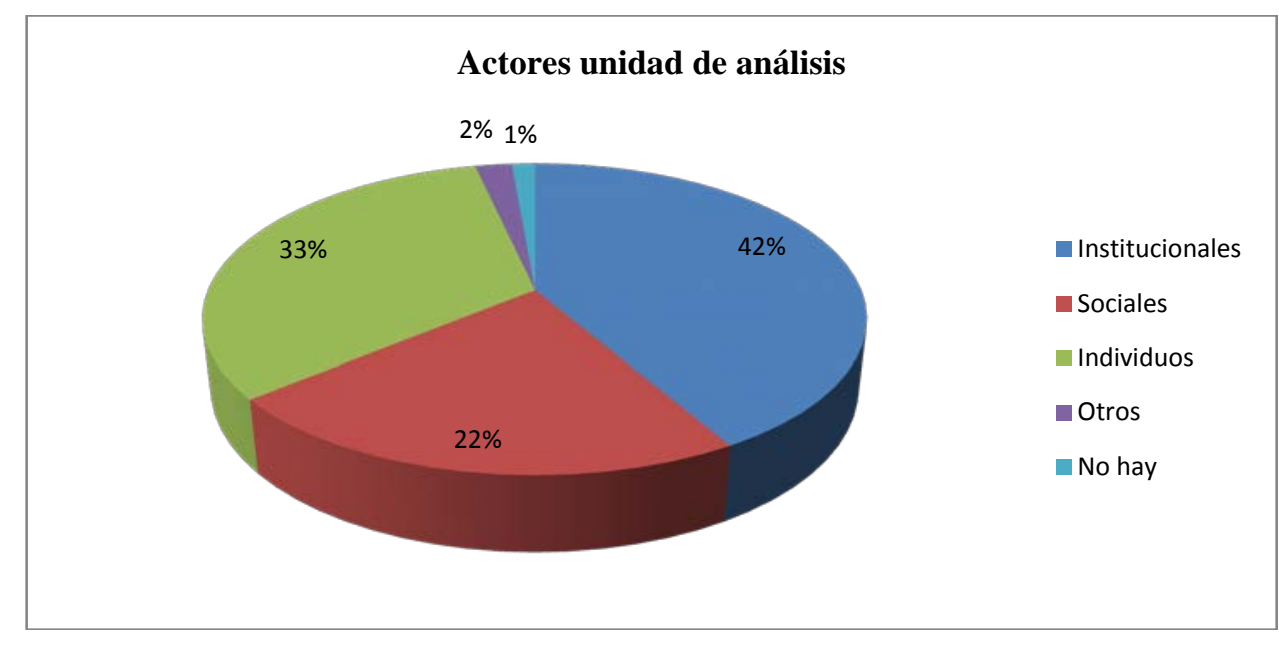

Gráfico 16

Fuente: elaboración propia a partir de los datos extraídos y analizados en la investigación

Estos resultados informan que son los actores institucionales los más referenciados. Esta circunstancia es similar a otras investigaciones que hemos realizado con esta metodología. Aquí lo que sí es diferente, y nos llama la atención, es el alto porcentaje de individuos como actores destacados de las informaciones. También señalamos que respecto a investigaciones anteriores, el porcentaje de actores sociales ha aumentado.

La agenda temática en la prensa de los países mediterráneos, la aportación de derechos y la creación de estados de opinión

También es de interés conocer quiénes son estos actores y con qué status son reconocidos y el rol que desempeñan.

En el gráfico presentado a continuación observamos que para los actores institucionales los resultados son los siguientes: 


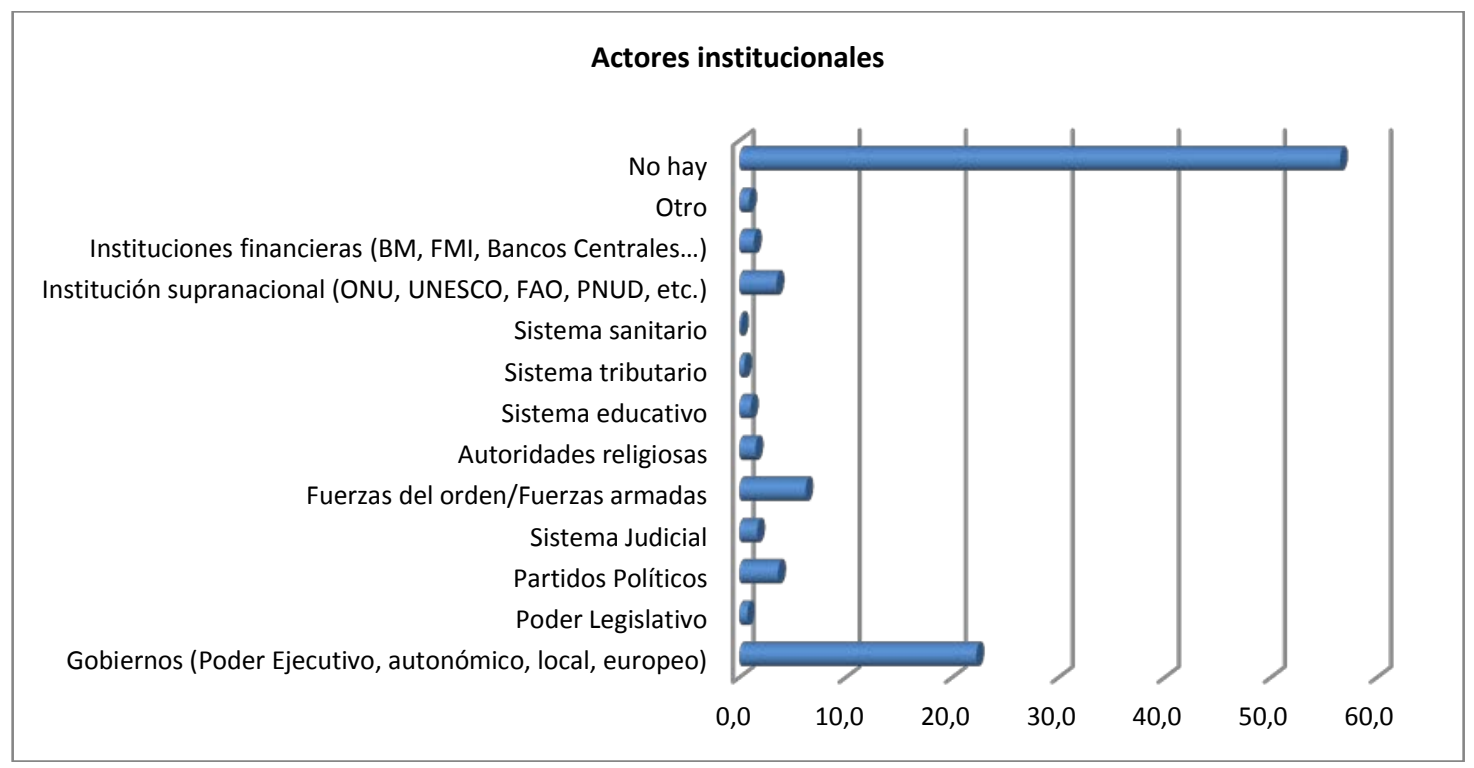

\section{Gráfico 17}

Fuente: elaboración propia a partir de los datos extraídos y analizados en la investigación

Por peso porcentual los actores vinculados a los gobiernos son los que cuentan con mayor presencia (22,3\%), seguido de las fuerzas del orden/fuerzas armadas (6,2\%). Mención de interés son las instituciones supranacionales (3,5\%)

En cuanto a los actores sociales veamos los resultados

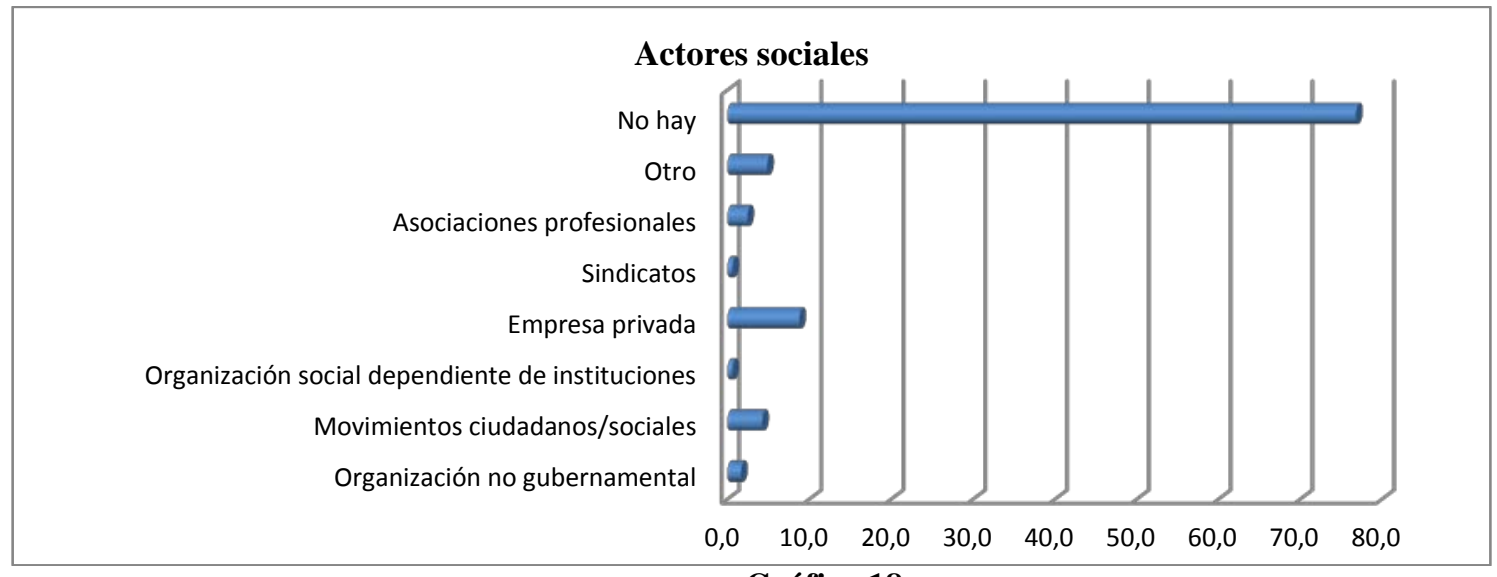

\section{Gráfico 18}

Fuente: elaboración propia a partir de los datos extraídos y analizados en la investigación Ricardo Carniel y Teresa Velázquez

Vemos que destacan la empresa privada (8,8\%), seguido de Movimientos sociales $(4,2 \%)$ y asociaciones profesionales $(2,4 \%)$ y muy poco para las ONG $(1,7 \%)$.

Entendemos que la presencia de empresas privadas tiene correlación con el tipo de relaciones multilaterales y bilaterales que henos destacado en el apartado anterior.

Respecto a los individuos como actores, veamos los resultados obtenidos 


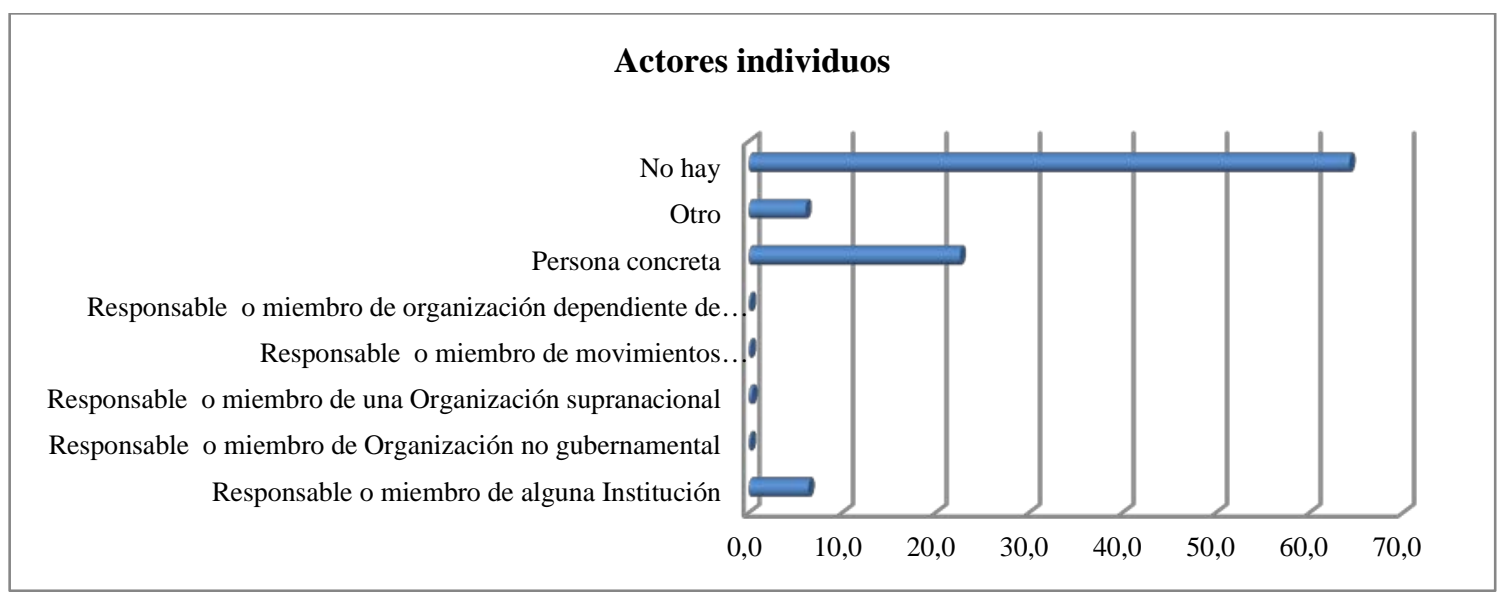

Gráfico 19

Fuente: elaboración propia a partir de los datos extraídos y analizados en la investigación

La individualización es la característica principal de esta variable. La presencia de individuo como persona concreta supone un $22,6 \%$ del total, frente a un $6,4 \%$ que se corresponde con responsable o miembro de alguna institución.

\section{CONCLUSIONES}

\section{El espacio intermediterráneo en los diarios objeto de estudio.}

La muestra seleccionada para esta investigación, y respecto al espacio intermediterráneo, indica que los diarios de los países árabes dedican más espacio a este ámbito. De las 660 unidades de análisis, 395 corresponden a los diarios de los países árabes, mientras que 265 se corresponden a los diarios europeos.

\section{La toma de decisión en los diarios analizados.}

Las informaciones sobre la Integración Intermediterránea no se encuentran mayoritariamente destacadas en los medios analizados y durante el período estudiado. El resultado de un $85,5 \%$ de los casos así lo indica.

Otro aspecto que redunda en esta afirmación es que en un 66,6\% de los casos, las informaciones ocupan hasta un 25\% de una página.

Sin embargo, sí que aparecen en la mitad superior de la página, en un 49\%, lo que indica que hasta cierto punto, y respecto a la localización, los medios conceden cierta importancia a las informaciones sobre este tema. 
La noticia es el género informativo más utilizado para referirse al tema. Un 83\% responde al género en el que los medios han narrado los aspectos vinculados al espacio intermediterráneo.

Destaca, también que la sección en la que se ubican las informaciones es la de Internacional, con un $40 \%$ de los casos.

Respecto a las fuentes utilizadas, mayoritariamente son propias (65\%) lo que indica que los medios de comunicación cuentan con recursos para cubrir este ámbito.

\section{La agenda temática y el espacio intermediterráneo}

Los resultados obtenidos en este estudio indican que los temas vinculados al espacio Intermediterráneo que interesan a los medios de comunicación se centran en Política y legislación, Paz y seguridad y Economía y finanzas. Son los temas más recurrentes.

Llama la atención que el enfoque mayoritariamente es de problema, lo que indica que este espacio es un espacio de conflicto.

Esta conclusión la observamos también en lo referente a Paz y seguridad en la que los valores más destacados se corresponden con conflicto armado, terrorismo internacional, delincuencia organizada, aunque hay un esperanzador valor sobre promoción de la paz y la convivencia.

Si la agenda temática se relaciona con la contribución de los medios de comunicación a la construcción social de la realidad, a partir de los resultados obtenidos en esta investigación vemos que la construcción mayoritaria da lugar al conflicto en este espacio hecho que, una vez más pone en evidencia aquellas intenciones de las organizaciones supracionales sobre "construir un espacio de paz y convivencia”

Asimismo, cuando se trata de referenciar hechos noticiables referidos a Derechos Humanos, la información más recurrente es la que se relaciona con violación y vulneración de los mismos.

Esto indica que los estados de opinión que se hayan podido generar durante el periodo analizado y sobre la integración intermediterránea estarán más abocados al una construcción simbólica de conflicto que de convivencia.

\section{Las relaciones multilaterales y bilaterales en el espacio intermediterráneo.}

Aquí destacamos las relaciones Norte-Sur en los ámbitos Políticos y de Paz y seguridad como pueda ser el Derecho internacional y derechos humanos, Delincuencia organizada, Migración irregular, Conflicto diplomático, Terrorismo nacionalista/independentista, Cooperación militar y/o policial, Promoción de la paz y la convivencia; o en relación a 
Economía y finanzas, donde destaca la Zona Euromediterránea de libre comercio los Recursos energéticos, Infraestructuras y transporte y alguna mención a Cooperación al desarrollo.

\section{Los actores presentes en las unidades de análisis}

En este aspecto, los actores institucionales son los que cuentan con una mayor presencia. Siendo los gobiernos los que aparecen más referenciados. En cuanto a los actores sociales los más destacados son asociados a la empresa privada. Respecto al actor individuo, la mención a persona concreta es la más destacada.

\section{BIBLIOGRAFIA}

GROSSI, G., La RAI sotto analisi : 1977-1980 : le ricerche promosse dalla verifica programmi tresmessi. Edizione Radiotelevisione Italiana, Turín, 1984

GROSSI, G., La Opinión pública: teoría del campo demoscópico, CIS, Madrid, CIS, 2007

McCOMBS, M. E., Setting the agenda : the mass media and public opinion, $2^{\mathrm{a}}$ edoción, Polity, Cambridge; Malden, Mass.

McCOMBS, M. E. y SHAW, D., "The agenda-setting function of mass media", Public Opinion Quarterly, 36 (2)/1972, pp. 176 y ss.

MARLETTI, C., Prima e dopo: tematizzazione e comunicazione política, ERI, Turín, 1985

ROSITI, F., I Modi dell'argomentazione e l'opinione pubblica, ERI, Turín, 1982

TUCHMAN, G., La Producción de la noticia: estudio sobre la construcción de la realidad, Gustavo Gili Barcelona, 1983

WOLF, Mauro, Los Efectos sociales de los media, Editorial Paidós Ibérica Barcelona, 1994 